\title{
Acknowledgement to Reviewers of Diagnostics in 2017
}

Diagnostics Editorial Office

MDPI AG, St. Alban-Anlage 66, 4052 Basel, Switzerland

Published: 10 January 2018

Peer review is an essential part in the publication process, ensuring that Diagnostics maintains high quality standards for its published papers. In 2017, a total of 57 papers were published in the journal. Thanks to the cooperation of our reviewers, the median time to first decision was 25 days and the median time to publication was 61 days. The editors would like to express their sincere gratitude to the following reviewers for their time and dedication in 2017:

Adhikari, Prakash

Akif, Turna

Almerich-Silla, José Manuel

Amarasena, Najith

Andreoni, Giuseppe

Banitsas, Konstantinos

Bankhead, Clare

Baptista, Pedro Viana

Becker, Therese $\mathrm{M}$.

Biermann, Martin

Bisharat, Naiel

Boisselier, Elodie

Bowen, Deborah J.

Bricout, Marine

Cai, Lisheng

Carpenter, Guy

Catry, Boudewijn

Cavoretto, Paolo

Cehofski, Lasse

Chatterjee, Jhinuk

Chen, Ruei-ming

Chen, Zhihang

Chiu, Ka Fung Peter

Combes, Jean-Damien

Coombs, Robert W.

Craven, Michael P.

De Biase, Dario

Delabaere, Amélie

Di Bartolomeo, Antonio

Dimitrakopoulou-Strauss, Antonia

Dorward, Jienchi

Eedunuri, Vijay Kumar

Ehrnhoefer, Dagmar

Ekpo, Ernest U.
Elgazzar, Abdelhamid H.

Fasano, Fabrizio

Ferrante, Mark A.

Fleischer, Arthur C.

Francis, Susan

Franklin, Gary M.

Giubileo, Filippo

Glaser, Gretchen E.

Gordon, Catherine A.

Hambrock, Thomas

Han, Seung Hee

Hendrikse, Harry

Hingorani, Dina

Holland, Katharina

Hsieh, Kuangwen

Islam, Kamrul

Iwata, Junichi

Jakowski, Joseph D.

Jeong, Keun-Yeong

Jia, Yinnong

Jiménez-Bonilla, Julio Francisco

Jin, Yan

Kanoun, Salim

Kantarci, Alpdogan

Kara, H. Volkan

Kaushik, Ajeet

Koshiyama, Masafumi

Kubina, Robert

La Rocca, Renato V.

Lakhal-Chaieb, Lajmi

Lamb, Benjamin W.

Lång, Kristina

Lee, Kwan Hyi

Lee, Zhenghong 
Leitman, I. Michael

Leung, Felix

Lewis, Mark

Li, Xiang-Guo

Licitra, Lisa

Liu, Shing-Hong

Liu, Xiaoke

Liu, Yu

Løbner-Olesen, Anders

López, Carmen Blanco

Lum, Ying Wei

Mariño, Inés $P$.

Marottoli, Richard A.

Marra, Paolo

Mashamba-Thompson, Tivani P.

Mayo, Ray

Miederer, Matthias

Milano, Francesco

Miles, Anne

Mirza, Aleem K.H.

Mora-Gutiérrez, José María

Morbelli, Silvia

Muyldermans, Serge

Mystkowska, Joanna

Narod, Steven A.

Nyström, Pär

Oćwieja, Magdalena

Okeke, Nwora Lance

Okumura, Nobuaki

Orsi, Andrea

Park, Mi-Suk

Parnetti, Lucilla

Piersma, Dafne

Pinsky, Paul F.

Polanec, Stephan

Pond, Dimity

Povlsen, Sebastian

Prabhulkar, Shradha

Priefer, Ronny

Pulford, Karen

Ramani, Vijay

Ramaraj, Pandurangan

Rispoli, Joseph

Ritter, Kerstin

Robert, Lidia

Rogerson, Stephen J.

Rostaing, Lionel

Sachpekidis, Christos

Sai, Kiran Kumar Solingapuram
Saini, Shikha

Saponara, Sergio

Sarkozy, Clémentine

Sathekge, Mike

Schachter, Steven C.

Schelhaas, Sonja

Schoder, Heiko

Schrum, Adam

Seiler, Stephen

Shea, Christopher M.

Shieh, Tzong-Ming

Singer, Eric A.

Singh, Virtaj

Smith, Heather F.

Soh, Jun Hui

Spanakis, Emmanouil G.

Subhi, Yousif

Sun, Meng

Sundar, Sudha

Szubert, Sebastian

Takebayashi, Shigeo

Tanaka, Yumiko Oishi

Temkin, Sarah

Thompson, Trevor

Tsai, Fuu-Jen

Ulum, Mokhamad Fakhrul

Uusi-Rasi, Kirsti

Vanhomwegen, Jessica

Vashist, Sandeep Kumar

Venkatesh, Alagarswamy G.

Venturella, Roberta

Viggiano, Davide

Vougas, Kostas

Warwick, Jane

Wei, Changyong

Weiergräber, Marco

Whelan, Rebecca J.

Wilczek, Brigitte

Willmann, Jürgen K.

Woods, Christopher Wildrick

$\mathrm{Xu}$, Wang

Yakobson, Boris A.

Yeh, Chih-ko

You, Xiaona

Zanello, Alessandro

Zhang, Ming

Zhu, Quing

Zhu, Zhaohui 